\title{
Biaxial versus uniaxial nematic stability in asymmetric rod-plate mixtures
}

\author{
H. H. Wensink, G. J. Vroege, ${ }^{*}$ and H. N. W. Lekkerkerker \\ Van't Hoff Laboratory for Physical and Colloid Chemistry, Debye Institute, Utrecht University, Padualaan 8 , \\ 3584 CH Utrecht, The Netherlands
}

(Received 30 May 2002; published 30 October 2002)

\begin{abstract}
The isotropic-nematic phase behavior of a binary mixture of rodlike and platelike particles is studied within Onsager's second virial theory. The phase behavior is obtained from the numerically exact equilibrium orientational distribution functions for both uniaxial and biaxial nematic phases. Inspired by recent experimental work on these systems we concentrated on asymmetric mixtures in which the excluded volume between the plates $v_{\mathrm{ex}}^{\mathrm{pp}}$ is larger than that between the rods $v_{\mathrm{ex}}^{\mathrm{rr}}$. Starting from the symmetric case $\left(v_{\mathrm{ex}}^{\mathrm{pp}} / v_{\mathrm{ex}}^{\mathrm{rr}}=1\right)$ and increasing the rod-plate excluded volume ratio we scrutinized the phase behavior, in particular focusing on the stability of the biaxial nematic phase. We observe that, at a certain asymmetry, the characteristic bicritical point is replaced by a two-phase region marking first order isotropic-biaxial transitions. Increasing the asymmetry even further leads to several demixing scenarios. First, there is a uniaxial-biaxial $\left(N^{+}-B\right)$ demixing scenario with an associated isotropic-uniaxial-biaxial $\left(I-N^{+}-B\right)$ triple equilibrium. Second, a uniaxial-uniaxial $\left(N^{+}-N^{-}\right)$demixing occurs in case of strongly asymmetric mixtures indicating that the biaxial nematic phase may become fully metastable. Since all predicted demixing scenarios lie in the experimentally accessible regime, there is a possibility of finding biaxial nematic structures in lyotropic colloidal rod-plate mixtures.
\end{abstract}

DOI: 10.1103/PhysRevE.66.041704

PACS number(s): 64.70.Md, 64.60.Cn, 61.30.Cz

\section{INTRODUCTION}

Since the pioneering work of Zocher and Langmuir [1,2], it has been known that dispersions of highly anisometrical rodlike or platelike colloidal particles exceeding a certain concentration undergo an orientational order-disorder transition from an isotropic state $(I)$, in which the particles are randomly oriented to an orientationally ordered nematic state $(N)$. Onsager [3] first showed that the phase transition can be explained on the basis of purely repulsive interactions between the particles. In his seminal work, he explained the phase transition as the result of a competition between orientational entropy which favors the isotropic state and the entropy effect associated with the orientation-dependent excluded volume of the anisometrical particles which favors the ordered nematic state. Onsager's theoretical approach, which was originally inspired by experimental observations of phase separating systems of pure colloidal rods (tobacco mosaic virus) [4] and platelets [2], can be extended to allow phase diagram calculations for binary mixtures of anisometric particles, e.g., rods with different lengths [5] or mixtures of rodlike and platelike particles [6]. The phase behavior of the latter systems is particularly interesting due to the possibility of having nematic phases with different symmetries, i.e., two uniaxial ones (a rod-rich $N^{+}$phase and a platedominated $N^{-}$phase) and a biaxial $B$ phase, in which rods and platelets are oriented along mutually perpendicular directors. An important parameter that governs the overall topology of the isotropic-nematic phase diagram is the ratio of the excluded volumes between two platelets and that between two rods, $v_{\mathrm{ex}}^{\mathrm{pp}} / v_{\mathrm{ex}}^{\mathrm{rr}}$, defining the asymmetry of the mixture. Setting this ratio equal to unity will produce phase diagrams that are symmetric about mole fraction $x=1 / 2$ (i.e.,

*FAX: +31-302533870. Email address: G.J.Vroege@chem.uu.nl equal portions of rods and plates), at least within a second virial approach. Henceforth, we will refer to these systems as symmetric mixtures. In general, asymmetric phase diagrams will be obtained when the excluded volume ratio is chosen to be larger or smaller than unity (asymmetric mixtures) or when higher virial terms are incorporated explicitly (e.g., in a computer simulation).

Previous theoretical studies on rod-plate mixtures, which have mainly focused on symmetric mixtures (for which $\left.v_{\mathrm{ex}}^{\mathrm{pp}} / v_{\mathrm{ex}}^{\mathrm{rr}}=1\right)$, can be subdivided into two groups. On the one hand, Onsager-type theories [6-10] were adopted allowing for a continuous treatment of both the positional and orientational degrees of freedom. On the other hand, mean-field lattice models $[11,12]$ were used in which the positional and/or orientational coordinates are discretized, such as the Zwanzig model [13] where the particle orientations are restricted to lie on one of the Cartesian axes. All theories predict the same qualitative behavior for the symmetric case; a stable biaxial nematic phase exists in between the rod- and plate-dominated uniaxial phases, and meets the isotropic phase in a bicritical point. However, van Roij and Mulder [12] showed that the biaxial nematic phase in a mixture of rectangular rodlike and platelike blocks, treated within a Zwanzig second virial theory, may become unstable with respect to demixing into the uniaxial nematic phases at some critical rod-plate excluded volume ratio. Computer simulations by Camp et al. [14] on symmetric mixtures of hard prolate and oblate ellipsoids confirmed that demixing can occur. Their phase diagrams, which were not symmetric due to the effect of higher-order particle interactions, essentially revealed a two-step demixing scenario where the biaxial nematic phase demixes into the uniaxial phases upon compression via a transitional plate-uniaxial-biaxial demixing region.

Experiments [15] on strongly asymmetric mixtures $\left(v_{\mathrm{ex}}^{\mathrm{pp}} / v_{\mathrm{ex}}^{\mathrm{rr}} \gg 1\right)$ using rod- and plate-shaped colloids (both with 
an aspect ratio of about 15) also showed a demixing into fractionated rod- and plate-dominated nematic phases, both probably having a uniaxial symmetry. In a previous paper [16], we were able to reproduce most features of the lowconcentration part of the experimental phase diagram in the context of the Onsager theory incorporating higher-order particle correlations with the Parsons rescaling approach. However, as we focused on the uniaxial nematic phases, using Gaussian trial functions to describe the equilibrium particle orientations in these phases, we did not explore the possibility of biaxial solutions in that study.

In this paper, we use the same model in the context of Onsager's second virial theory but we now explicitly include the possibility of biaxial symmetry by performing the exact free energy minimization with respect to the orientational degrees of freedom and solving the resulting integral equations exactly, using numerical schemes. In this way we obtain the numerically exact orientational distribution functions (ODFs) for the aligned phases without having to rely on approximations such as using trial ODFs with a predescribed form [16], discretized orientation models [11,12], or the socalled L2 model $[6,9,10]$. In the latter case, the excluded volumes are represented as a series expansion in terms of spherical harmonics truncated after the first term, which is only reliable for very weakly aligned nematic phases. Since the phase behavior of anisometric particles, in general, depends crucially on the approximations used in the description of the excluded volume interactions (see, e.g., Refs. [10,17]), the most credible results will be obtained when the excluded volume integrals are solved exactly, i.e., without approximations.

In this paper we examine the effect of the asymmetry, induced by increasing the rod-plate excluded volume ratio from unity, on the phase behavior of rod-plate mixtures. Our main interest is to establish possible phase diagram scenarios for such mixtures. In particular, we focus on the stability of the biaxial nematic phase.

This paper is structured as follows. In Sec. II A we give a short description of the Onsager theory in the specific case of binary mixtures of rods and plates. The numerical techniques used to solve the minimization equations and to calculate the entropy integrals are outlined in Sec. II B. In Sec. II C we introduce order parameters to distinguish between the liquid crystals phases. A bifurcation analysis is presented in Sec. II D, which we use to locate the onset of a new nematic symmetry out of a given reference phase. In Sec. IIE we discuss the criteria used to discriminate between stable and metastable phases. The phase diagrams will be presented in Sec. III and several scenarios are discussed in detail. Finally, some conclusions are made in Sec. IV.

\section{THEORY}

\section{A. Onsager theory}

We consider a binary mixture of hard cylindric rods and platelets in a macroscopic volume $V$. The particles involved are characterized by four parameters: the length $L_{r}$ and the diameter $D_{r}$ of the rods (with $L_{r}>D_{r}$ ) and the diameter $D_{p}$ and length (thickness) $L_{p}$ of the platelets (with $D_{p}>L_{p}$ ).
The details of the exact shape of the particles are found to be irrelevant for the general argument, provided that the particles are sufficiently anisometrical, i.e., $L_{r} / D_{r} \gg 1$ and $D_{p} / L_{p} \gg 1$. The Helmholtz free energy of the mixture in the Onsager [3] treatment is given by

$$
\frac{\beta F}{N}=\mathrm{const}+\ln c-1+\sum_{j=1,2} x_{j}\left[\ln x_{j}+\sigma_{j}\right]+c B_{2},
$$

where $\beta=\left(k_{B} T\right)^{-1}$ with $k_{B}$ Boltzmann's constant and $T$ the temperature. The irrelevant constant includes terms independent of the particle densities. Henceforth, we define $x_{2}=x$ as the mole fraction of the platelets. Furthermore, $c$ is the total dimensionless concentration, $c=b N / V$, with $b=\pi L_{r}^{2} D_{r} / 4$ the average excluded volume between two randomly oriented thin rods. The free energy (1) consists of several entropic contributions. Apart from an ideal and mixing contribution there is an orientational entropy involving the quantity $\sigma_{j}$, defined as

$$
\sigma_{j} \equiv \int f_{j}(\Omega) \ln \left[4 \pi f_{j}(\Omega)\right] d \Omega, \quad j=1,2 .
$$

Here, $f_{j}(\Omega)$ represents the ODF describing the distribution of the solid angle $\Omega$ of the $j$ th-particle's orientation vector. The ODF must be normalized according to $\int f_{j}(\Omega) d \Omega \equiv 1$. In the isotropic state, all orientations are equally probable, which implies $f_{\text {iso }} \equiv 1 / 4 \pi$ and $\sigma_{\text {iso }} \equiv 0$. In the nematic state, however, $\sigma$ will be larger than zero because $f_{j}(\Omega)$ is a peaked function.

The last term in Eq. (1) is the excluded volume entropy due to the repulsive interactions treated at the level of Onsager's second virial approximation. The second virial coefficient $B_{2}$ for a binary mixture of anisometric particles is defined as

$$
B_{2}=(1-x)^{2} \rho_{11}+2 x(1-x) q_{12} \rho_{12}+x^{2} q_{22} \rho_{22},
$$

where the parameters $\rho_{j k}$ represent the angular average of the excluded volume between particles of type $j$ and $k$ relative to their excluded volume in the isotropic phase,

$$
\rho_{j k} \equiv \iint \frac{v_{\mathrm{excl}}^{j k}(\gamma)}{v_{\mathrm{excl}, \mathrm{iso}}^{j k}} f_{j}(\Omega) f_{k}\left(\Omega^{\prime}\right) d \Omega d \Omega^{\prime},
$$

in which $\gamma$ is the angle between the particle orientation vectors. From this definition we immediately see that $\rho_{j k} \equiv 1$ in the isotropic phase, whereas $0<\rho_{j k}<1$ in the nematic state. For sufficiently anisometric particles the leading terms of the excluded volumes are given by [3]

$$
\begin{gathered}
v_{\mathrm{excl}}^{\mathrm{pp}}(\gamma) \sim \frac{\pi}{2} D_{p}^{3}|\sin \gamma|, \\
v_{\mathrm{excl}}^{\mathrm{rp}}(\gamma) \sim \frac{\pi}{4} L_{r} D_{p}^{2}|\cos \gamma|, \\
v_{\mathrm{excl}}^{\mathrm{rr}}(\gamma) \sim 2 L_{r}^{2} D_{r}|\sin \gamma| .
\end{gathered}
$$


Using the isotropic averages $\langle|| \sin \gamma \mid\rangle\rangle_{\text {iso }}=\pi / 4$ and $\langle\langle|\cos \gamma|\rangle\rangle_{\text {iso }}=1 / 2$ we obtain the following expressions for $\rho_{j k}$ :

$$
\begin{gathered}
\rho_{j j}=\frac{4}{\pi} \iint\left|\sin \gamma\left(\Omega, \Omega^{\prime}\right)\right| f_{j}(\Omega) f_{j}\left(\Omega^{\prime}\right) d \Omega d \Omega^{\prime}, \quad j=1,2, \\
\rho_{12}=2 \iint\left|\cos \gamma\left(\Omega, \Omega^{\prime}\right)\right| f_{1}(\Omega) f_{2}\left(\Omega^{\prime}\right) d \Omega d \Omega^{\prime} .
\end{gathered}
$$

The parameters $q_{12}$ and $q_{22}$ in Eq. (3) quantify the excluded volume between two randomly oriented particles (a rod and a platelet and two platelets, respectively) relative to that between two rods

$$
q_{12}=\frac{1}{4}\left(\frac{D_{p}}{D_{r}}\right)^{2} /\left(\frac{L_{r}}{D_{r}}\right) \quad \text { and } \quad q_{22}=\frac{\pi}{4}\left(\frac{D_{p}}{D_{r}}\right)^{3} /\left(\frac{L_{r}}{D_{r}}\right)^{2} \text {. }
$$

These parameters are very important since they determine the asymmetry of the rod-plate mixture. Setting $q_{22}$ equal to unity will render the free energy symmetric about $x=0.5$ (within the second virial approach) as we see from Eq. (3). Consequently, all phase diagrams must possess the same symmetric topology $[6,8,12]$. In our case, $q_{22}$ will generally be larger than unity (i.e., the isotropic excluded volume of the plates is larger than that of the rods), which implies that the symmetry is lost and all phase diagrams are asymmetric.

In order to calculate the parameters $\rho_{j k}$ and $\sigma_{j}$ we must determine the shape of the thermodynamic equilibrium ODF. This can be done by minimizing the free energy with respect to $f_{j}$ by performing a functional differentiation under the constraint of the normalization condition,

$$
\frac{\delta}{\delta f_{j}(\Omega)}\left\{\frac{\beta F}{N}+\lambda_{j}\left[1-\int f_{j}(\Omega) d \Omega\right]\right\}=0, \quad j=1,2,
$$

where $\lambda_{j}$ are the Lagrange undetermined multipliers which follow from the normalization conditions. This results in the following coupled set of Euler-Lagrange equations:

$$
\begin{aligned}
\lambda_{1}= & \ln \left[4 \pi f_{1}(\Omega)\right] \\
& +\frac{8 c}{\pi}(1-x) \int\left|\sin \gamma\left(\Omega, \Omega^{\prime}\right)\right| f_{1}\left(\Omega^{\prime}\right) d \Omega^{\prime} \\
& +4 c x q_{12} \int\left|\cos \gamma\left(\Omega, \Omega^{\prime}\right)\right| f_{2}\left(\Omega^{\prime}\right) d \Omega^{\prime}, \\
\lambda_{2}= & \ln \left[4 \pi f_{2}(\Omega)\right] \\
& +4 c(1-x) q_{12} \int\left|\cos \gamma\left(\Omega, \Omega^{\prime}\right)\right| f_{1}\left(\Omega^{\prime}\right) d \Omega^{\prime} \\
& +\frac{8 c}{\pi} x q_{22} \int\left|\sin \gamma\left(\Omega, \Omega^{\prime}\right)\right| f_{2}\left(\Omega^{\prime}\right) d \Omega^{\prime} .
\end{aligned}
$$

These two nonlinear integral equations constitute the starting formulas for the phase equilibria calculations in our work. Since there is no exact solution to the equations above we must adopt numerical techniques to obtain the equilibrium
ODF of the nematic phase at a given $x$ and $c$. This issue will be discussed in detail in the following paragraph.

Once the minimization problem has been solved, the compositions and concentrations of the coexisting phases can be found by imposing the standard conditions of equal osmotic pressure $\Pi$ and chemical potentials $\mu_{j}$,

$$
\begin{aligned}
b \beta \Pi & \equiv-b\left(\frac{\partial \beta F / N}{\partial V}\right)_{N_{1}, N_{2}, T} \simeq c+c^{2} B_{2}, \\
\beta \mu_{1} & \equiv\left(\frac{\partial \beta F / N}{\partial N_{1}}\right)_{N_{2}, V, T} \\
& \simeq \ln c+\ln (1-x)+\sigma_{1}+2 c\left[(1-x) \rho_{11}+x q_{12} \rho_{12}\right], \\
\beta \mu_{2} & \equiv\left(\frac{\partial \beta F / N}{\partial N_{2}}\right)_{N_{1}, V, T} \\
& \simeq \ln c+\ln x+\sigma_{2}+2 c\left[(1-x) q_{12} \rho_{12}+x q_{22} \rho_{22}\right] .
\end{aligned}
$$

Recall that $\sigma_{j} \equiv 0$ and $\rho_{j k} \equiv 1$ for the isotropic phase. The coexistence equations were solved using standard NewtonRaphson iteration. The accuracy in the mole fractions and concentrations were chosen to be at least five significant digits.

\section{B. Minimization of the free energy}

\section{Series expansion solution}

A systematic way to tackle the integral equations (9) is to expand the kernels $|\sin \gamma|$ and $|\cos \gamma|$ in terms of Legendre polynomials $P_{n}$. Following Kayser and Raveché [18] and Stroobants and Lekkerkerker [6] we write

$$
\begin{aligned}
& |\sin \gamma|=\frac{\pi}{4}+\sum_{n=1}^{\infty} d_{2 n} P_{2 n}(\cos \gamma), \\
& |\cos \gamma|=\frac{1}{2}+\sum_{n=1}^{\infty} c_{2 n} P_{2 n}(\cos \gamma),
\end{aligned}
$$

with coefficients [19]

$$
\begin{gathered}
d_{2 n}=-\frac{\pi(4 n+1)(2 n-3) ! !(2 n-1) ! !}{2^{2 n+2} n !(n+1) !}, \\
c_{2 n}=\frac{(-1)^{n+1}(4 n+1)(2 n-3) ! !}{2^{n+1}(n+1) !} .
\end{gathered}
$$

For symmetry reasons only even Legendre polynomials need be retained [8]. To include the possibility of biaxial symmetry we use the addition theorem of spherical harmonics [20] to rewrite $P_{2 n}(\cos \gamma)$ in terms of a bilinear expansion in $P_{2 n}(\cos \theta)$ and its associated Legendre polynomials $P_{2 n}^{m}(\cos \theta)$, 


$$
\begin{aligned}
P_{2 n}(\cos \gamma)= & P_{2 n}(\cos \theta) P_{2 n}\left(\cos \theta^{\prime}\right)+2 \sum_{m=1}^{2 n} \frac{(2 n-m) !}{(2 n+m) !} \\
& \times P_{2 n}^{m}(\cos \theta) P_{2 n}^{m}\left(\cos \theta^{\prime}\right) \cos m\left(\phi-\phi^{\prime}\right) .
\end{aligned}
$$

Here, $\theta$ is the polar angle between the particle orientation vector and the nematic director and $\phi$ is the azimuthal angle describing the orientation in the plane perpendicular to that director. Substituting Eqs. (11) and (13) into the integral equations (9) and some rearranging leads to

$$
\begin{aligned}
f_{j}(\Omega)= & Z_{j}^{-1} \exp \left[\sum _ { n = 1 } ^ { \infty } \left\{\alpha_{2 n}^{(j)} P_{2 n}(\cos \theta)\right.\right. \\
& \left.\left.+\sum_{m=1}^{n} k_{n m} \beta_{2 n}^{2 m(j)} P_{2 n}^{2 m}(\cos \theta) \cos 2 m \phi\right\}\right], \quad j=1,2
\end{aligned}
$$

where $k_{n m}=2(2 n-2 m) ! /(2 n+2 m) !$ and $Z_{j}$ is the normalization factor. For symmetry reasons, only even $-m$ associated Legendre functions need be included and all $\sin m \phi$ arising from the addition theorem vanish [8]. The coefficients $\alpha_{2 n}^{(j)}$ and $\beta_{2 n}^{2 m(j)}$ are given by

$$
\begin{aligned}
& \alpha_{2 n}^{(1)}=-2 c\left[(1-x) \frac{4}{\pi} d_{2 n}\left\langle P_{2 n}\right\rangle_{f_{1}}+2 x q_{12} c_{2 n}\left\langle P_{2 n}\right\rangle_{f_{2}}\right], \\
& \alpha_{2 n}^{(2)}=-2 c\left[2(1-x) q_{12} c_{2 n}\left\langle P_{2 n}\right\rangle_{f_{1}}+x \frac{4}{\pi} q_{22} d_{2 n}\left\langle P_{2 n}\right\rangle_{f_{2}}\right],
\end{aligned}
$$

and

$$
\begin{aligned}
\beta_{2 n}^{2 m(1)}= & -2 c\left[(1-x) \frac{4}{\pi} d_{2 n}\left\langle P_{2 n}^{2 m} \cos 2 m \phi\right\rangle_{f_{1}}\right. \\
& \left.+2 x q_{12} c_{2 n}\left\langle P_{2 n}^{2 m} \cos 2 m \phi\right\rangle_{f_{2}}\right]
\end{aligned}
$$

$$
\begin{aligned}
\beta_{2 n}^{2 m(2)}= & -2 c\left[2(1-x) q_{12} c_{2 n}\left\langle P_{2 n}^{2 m} \cos 2 m \phi\right\rangle_{f_{1}}\right. \\
& \left.+x \frac{4}{\pi} q_{22} d_{2 n}\left\langle P_{2 n}^{2 m} \cos 2 m \phi\right\rangle_{f_{2}}\right] .
\end{aligned}
$$

The values of these coefficients are found by numerically solving the following coupled consistency equations:

$$
\begin{gathered}
\left\langle P_{2 n}\right\rangle_{f_{j}}=\int f_{j}(\Omega) P_{2 n}(\cos \theta) d \Omega, \quad n=1,2, \ldots, N, \\
\left\langle P_{2 n}^{2 m} \cos 2 m \phi\right\rangle_{f_{j}}=\int f_{j}(\Omega) P_{2 n}^{2 m}(\cos \theta) \cos 2 m \phi d \Omega, \\
n, m=1,2, \ldots, N \quad(m \leqslant n) .
\end{gathered}
$$

Note that $d \Omega=d(\cos \theta) d \phi$. Assuming the expansion in Eq. (14) to converge after a finite number of terms, we truncate the series after the $N$ th term. In case of uniaxial symmetry, the biaxial coefficients $\beta_{2 n}^{2 m(j)}$ are zero, which means that we only have to solve the set of $2 N$ consistency equations (17) using Eqs. (14) and (15). In case of biaxial symmetry, however, both sets, Eqs. (17) and (18), must be solved simultaneously, which implies solving $N(N+1)$ equations iteratively. Obviously, the number of $N$ depends on the degree of alignment of the nematic phase via the mole fraction and concentration. Following Ref. [6] we chose $N=7$ as a minimum for weakly ordered nematic phases and we increased its value up to a maximum $N=12$ for higher concentrations. The numerical integrations were performed using Gaussian quadrature. The initial trial ODFs were those in the perfectly aligned uniaxial (or biaxial) nematic phase. The solutions were iterated until the normalization factors $Z_{j}$ had converged to within $10^{-6}$.

Once the consistency equations have been solved, the entropic contributions $\sigma_{j}$ and $\rho_{j k}$ can be calculated from

$$
\begin{aligned}
& \sigma_{j}=-\ln 4 \pi Z_{j}+\sum_{n=1}^{N}\left\{\alpha_{2 n}^{(j)}\left\langle P_{2 n}\right\rangle_{f_{j}}+\sum_{m=1}^{n} k_{n m} \beta_{2 n}^{2 m(j)}\left\langle P_{2 n}^{2 m} \cos 2 m \phi\right\rangle_{f_{j}}\right\}, \quad j=1,2, \\
& \rho_{j j}=1+\frac{4}{\pi} \sum_{n=1}^{N} d_{2 n}\left\{\left\langle P_{2 n}\right\rangle_{f_{j}}^{2}+\sum_{m=1}^{n} k_{n m}\left\langle P_{2 n}^{2 m} \cos 2 m \phi\right\rangle_{f_{j}}^{2}\right\}, \quad j=1,2, \\
& \rho_{12}=1+2 \sum_{n=1}^{N} c_{2 n}\left\{\left\langle P_{2 n}\right\rangle_{f_{1}}\left\langle P_{2 n}\right\rangle_{f_{2}}+\sum_{m=1}^{n} k_{n m}\left\langle P_{2 n}^{2 m} \cos 2 m \phi\right\rangle_{f_{1}}\left\langle P_{2 n}^{2 m} \cos 2 m \phi\right\rangle_{f_{2}}\right\} .
\end{aligned}
$$

\section{Direct numerical solution}

The main drawback of the series solution is that the convergence becomes very sluggish when the nematic phase is strongly aligned. To obtain reasonable quantitative results, $N$ should be taken very large $(N \gg 10)$ in that regime which makes the numerical procedure computationally awkward. 
To make headway, we may consider an alternative method, proposed by Herzfeld et al. [21], in which the coupled set of integral equations (9) is solved directly by assuming a grid of angles $\Omega$ and $\Omega^{\prime}$. Taking the exponentiated form of Eq. (9) and eliminating the Lagrange multipliers using the normalization conditions of the ODFs we may rewrite Eq. (9) in an iterative form,

$$
\begin{gathered}
f_{1}^{(n+1)}(\Omega)=\frac{\exp \left[-\frac{8 c}{\pi}(1-x) \int\left|\sin \gamma\left(\Omega, \Omega^{\prime}\right)\right| f_{1}^{(n)}\left(\Omega^{\prime}\right) d \Omega^{\prime}-4 c x q_{12} \int\left|\cos \gamma\left(\Omega, \Omega^{\prime}\right)\right| f_{2}^{(n)}\left(\Omega^{\prime}\right) d \Omega^{\prime}\right]}{\int d \Omega \exp \left[-\frac{8 c}{\pi}(1-x) \int\left|\sin \gamma\left(\Omega, \Omega^{\prime}\right)\right| f_{1}^{(n)}\left(\Omega^{\prime}\right) d \Omega^{\prime}-4 c x q_{12} \int\left|\cos \gamma\left(\Omega, \Omega^{\prime}\right)\right| f_{2}^{(n)}\left(\Omega^{\prime}\right) d \Omega^{\prime}\right]}, \\
f_{2}^{(n+1)}(\Omega)=\frac{\exp \left[-4 c(1-x) q_{12} \int\left|\cos \gamma\left(\Omega, \Omega^{\prime}\right)\right| f_{1}^{(n)}\left(\Omega^{\prime}\right) d \Omega^{\prime}-\frac{8 c}{\pi} x q_{22} \int\left|\sin \gamma\left(\Omega, \Omega^{\prime}\right)\right| f_{2}^{(n)}\left(\Omega^{\prime}\right) d \Omega^{\prime}\right]}{\int d \Omega \exp \left[-4 c(1-x) q_{12} \int\left|\cos \gamma\left(\Omega, \Omega^{\prime}\right)\right| f_{1}^{(n)}\left(\Omega^{\prime}\right) d \Omega^{\prime}-\frac{8 c}{\pi} x q_{22} \int\left|\sin \gamma\left(\Omega, \Omega^{\prime}\right)\right| f_{2}^{(n)}\left(\Omega^{\prime}\right) d \Omega^{\prime}\right]} .
\end{gathered}
$$

The integrations over the solid angles $\Omega^{\prime}$ were carried out by Simpson's quadrature. We considered intervals of $[0, \pi / 2]$ for the polar angle $\theta$ and $[0,2 \pi]$ for the azimuthal angle $\phi$. The intervals were discretized into $J_{\theta}$ and $J_{\phi}$ equal parts. For the uniaxial nematic phases, the integrations over the azimuthal angle vanish so that we need only perform numerical integrations over the polar angle. Accurate results for highly ordered uniaxial nematic phases were obtained using $J_{\phi}$ $=1000$ and $J_{\theta}=400$. Refining the grid size even further did not lead to significant changes in the $\rho_{j k}$ and $\sigma_{j}$ reported here. Initial guesses for $f_{1}(\Omega)$ and $f_{2}(\Omega)$ were used to solve the coupled set (20) iteratively. The solutions were iterated until the convergence criterion $\max \left|f_{j}^{(n+1)}(\Omega)-f_{j}^{(n)}(\Omega)\right|$ $<10^{-8}(j=1,2)$ was satisfied. Once the equilibrium ODFs were obtained, the entropic contributions $\sigma_{j}$ and $\rho_{j k}$ could be calculated straightforwardly from Eqs. (2) and (6) using Simpson's quadrature.

\section{Order parameters}

In order to identify the isotropic and nematic phases, we introduce uniaxial $\left(S_{j}\right)$ and biaxial $\left(\Delta_{j}\right)$ order parameters for each component $j$. Following Ref. [6] we define

$$
\begin{gathered}
S_{j}=\left\langle P_{2}(\cos \theta)\right\rangle_{f_{j}}=\frac{1}{2}\left\langle 3 a_{z}^{2}-1\right\rangle_{f_{j}}, \\
\Delta_{j}=\frac{1}{3}\left\langle P_{2}^{2}(\cos \theta) \cos 2 \phi\right\rangle_{f_{j}}=\left\langle a_{x}^{2}\right\rangle_{f_{j}}-\left\langle a_{y}^{2}\right\rangle_{f_{j}},
\end{gathered}
$$

where $a_{s}$ is the projection of the particle orientation vector onto the $s$ axis of the reference frame. Thus $S_{j}$ describes the ordering of the axes of the rods and plates with respect to the $z$ axis whereas $\Delta_{j}$ describes the ordering of the species in the $x-y$ plane. For random orientations (isotropic phase) $\left\langle a_{s}^{2}\right\rangle$ $=1 / 3$ (with $s=x, y, z$ ), so that all order parameters are zero. In case of uniaxial order, the biaxial order parameters $\Delta_{j}$ are zero because there is no preferred direction in the $x-y$ plane $\left(\left\langle a_{x}^{2}\right\rangle=\left\langle a_{y}^{2}\right\rangle=0\right)$. In case of biaxial symmetry all order parameters will generally be nonzero.

When $0<S_{j} \leqslant 1$, the particle orientation vectors of component $j$ are preferentially oriented along the $z$ axis (polar alignment) whereas a negative value $\left(-0.5 \leqslant S_{j}<0\right)$ indicates that the particles lie preferentially in the $x-y$ plane (planar alignment). In the actual calculations we used two types of reference frames; a rod $\left(\mathrm{N}^{+}\right)$reference frame in which the rods point along the $z$ axis and the platelets' normal vectors lie in the $x-y$ plane and, second, a plate $\left(N^{-}\right)$reference frame in which the $z$ axis is oriented along the preferred direction of the plates' normal vector in a discotic phase, while the rods are oriented in the $x-y$ plane. Since the $N^{+}$ phase is characterized by polar alignment of the rods and planar alignment of the platelets we have $S_{R}>0$ and $S_{P}<0$ (within the rod reference frame). In the $N^{-}$phase, the situation is reversed so that $S_{P}>0$ and $S_{R}<0$ (within the plate reference frame).

\section{Bifurcation analysis}

\section{Isotropic-uniaxial nematic bifurcation}

The isotropic ODF $f_{j} \equiv 1 / 4 \pi$ is a trivial solution to Eqs. (9) for any concentration and mole fraction. At higher concentrations, however, the forms of the equilibrium ODFs will contain orientation dependent contributions indicating anisotropic phase solutions. These solutions will continuously split off from the isotropic branch at the $I-N$ bifurcation point. To find this point, we may assume that close to the $I-N$ bifurcation the nematic order is vanishingly small. Retaining only the first Legendre polynomial in Eqs. (11) and (14) and linearizing with respect to the coefficient $\alpha_{2}^{(j)}$ gives

$$
f_{j}(\theta)=\frac{1}{4 \pi}\left[1+\alpha_{2}^{(j)} P_{2}(\cos \theta)\right], \quad j=1,2 .
$$

Substituting this into the consistency equations (17) yields $\left\langle P_{2}\right\rangle_{f_{j}} \equiv S_{j}=\alpha_{2}^{(j)} / 5$. Consequently, the coefficients (15) for the uniaxial nematic phases read 


$$
\begin{aligned}
& \alpha_{2}^{(1)}=\frac{c}{4}\left[(1-x) \alpha_{2}^{(1)}-2 x q_{12} \alpha_{2}^{(2)}\right], \\
& \alpha_{2}^{(2)}=\frac{c}{4}\left[-2(1-x) q_{12} \alpha_{2}^{(1)}+x q_{22} \alpha_{2}^{(2)}\right] .
\end{aligned}
$$

These equations yield (for a given mole fraction $x$ ) the bifurcation concentration as the root of the characteristic equation $\operatorname{det} \mathbf{M}=0$, where

$$
\mathbf{M}=\left(\begin{array}{cc}
1-\frac{c}{4}(1-x) & \frac{c}{2} x q_{12} \\
\frac{c}{2}(1-x) q_{12} & 1-\frac{c}{4} x q_{22}
\end{array}\right) .
$$

The characteristic equation thus reads

$$
1-\frac{c}{4}\left[(1-x)+x q_{22}\right]+\left(\frac{c}{4}\right)^{2}\left[x(1-x)\left(q_{22}-4 q_{12}^{2}\right)\right]=0 .
$$

The concentration at which a bifurcation from the isotropic to a uniaxial nematic phase can be expected is given by the lowest positive solution of Eq. (25).

\section{Uniaxial-biaxial nematic bifurcation}

The same analysis described above can be adopted to locate the onset of biaxial order from a uniaxial reference phase. Assuming the lowest order of biaxiality $(m=1)$ in Eq. (14) and linearizing with respect to $\beta_{2}^{2(j)}$, we may write the biaxial solution close to the $N-B$ bifurcation point as follows:

$$
\begin{aligned}
f_{j}(\Omega)= & Z_{j}^{-1} \exp \left[\sum_{n=1}^{\infty} \alpha_{2 n}^{(j)} P_{2 n}(\cos \theta)\right] \\
& \times\left[1+\sum_{n=1}^{\infty} k_{n 1} \beta_{2 n}^{2(j)} P_{2 n}^{2}(\cos \theta) \cos 2 \phi\right] \\
= & f_{j}^{N}(\theta)\left[1+\sum_{n=1}^{\infty} k_{n 1} \beta_{2 n}^{2(j)} P_{2 n}^{2}(\cos \theta) \cos 2 \phi\right], \quad j=1,2,
\end{aligned}
$$

where $f_{j}^{N}(\theta)$ is the ODF of the uniaxial reference phase. Inserting Eq. (26) into Eq. (18) yields

$$
\left\langle P_{2 n}^{2} \cos 2 \phi\right\rangle_{f_{j}}=\sum_{k=1}^{\infty} \beta_{2 k}^{2} W_{n k}^{(j)}
$$

with

$$
\begin{aligned}
W_{n k}^{(j)} & =\frac{(2 k-2) !}{(2 k+2) !}\left\langle P_{2 n}^{2}(\cos \theta) P_{2 k}^{2}(\cos \theta)\right\rangle_{f_{j}^{N}(\theta)} \\
& =\frac{(2 k-2) !}{(2 k+2) !} \int_{0}^{1} P_{2 n}^{2}(\cos \theta) P_{2 k}^{2}(\cos \theta) f_{j}^{N}(\theta) d(\cos \theta) .
\end{aligned}
$$

Inserting Eq. (27) into the biaxial coefficients (16) we obtain the following linear set:

$$
\begin{aligned}
\beta_{2 n}^{2(1)}= & c \sum_{k=1}^{N}\left\{\left[-\frac{8}{\pi}(1-x) d_{2 n} W_{n k}^{(1)}\right] \beta_{2 k}^{2(1)}\right. \\
& \left.-\left[4 x q_{12} c_{2 n} W_{n k}^{(2)}\right] \beta_{2 k}^{2(2)}\right\}, \\
\beta_{2 n}^{2(2)}= & c \sum_{k=1}^{N}\left\{\left[-4(1-x) q_{12} c_{2 n} W_{n k}^{(1)}\right] \beta_{2 k}^{2(1)}\right. \\
& \left.-\left[\frac{8}{\pi} x q_{22} d_{2 n} W_{n k}^{(2)}\right] \beta_{2 k}^{2(2)}\right\} .
\end{aligned}
$$

When we truncate the series after the $N$ th term, the characteristic determinant $\mathbf{M}$ for this set is a $2 N \times 2 N$ matrix. It is convenient to rewrite the matrix $\mathbf{M}$ in the form $\mathbf{I}-c \mathbf{A}$, where $\mathbf{I}$ is the unit matrix and $\mathbf{A}$ is a numerical matrix. The characteristic equation is then given by

$$
\operatorname{det} \mathbf{M}=\operatorname{det}[\mathbf{I}-c \mathbf{A}]=\operatorname{det}\left[\mathbf{A}-c^{-1} \mathbf{I}\right]=0 .
$$

The bifurcation concentration is found by numerically determining the eigenvalues of the matrix $\mathbf{A}$. The concentration at which a bifurcation from a uniaxial to a biaxial symmetry can be expected is given by the inverse of the highest real, positive eigenvalue of $\mathbf{A}$. Since the parameters $W_{n k}^{(j)}$ in $\mathbf{A}$ are dependent on the concentration through the ODFs of the uniaxial nematic reference phase, the bifurcation points must be calculated self-consistently. The technique is to compute $W_{n k}^{(j)}[\mathrm{Eq} .(27)]$ for a given initial concentration (using either the series expansion method or a numerical grid, see Sec. II B) and then put it into the bifurcation equation (30) and find the desired root. For that concentration, new parameters $W_{n k}^{(j)}$ were calculated and inserted into Eq. (30) to find the new root. This procedure was repeated until the concentration had converged to within $10^{-6}$.

\section{E. Biaxiality and demixing}

As already mentioned in the Introduction, the central issue in our paper is to assess the stability of the biaxial nematic phase in relation to the mixture's asymmetry. It is important to realize that the biaxial nematic phase may be metastable with respect to some demixing transition, e.g., a phase separation into two uniaxial nematic phases $\left(N^{+}\right.$and $N^{-}$). In this respect, it is instructive to consider the Gibbs free energy, defined as

$$
\frac{\beta G}{N}=\frac{\beta F}{N}+c^{-1}(b \beta \Pi) .
$$

By calculating the Gibbs free energy as a function of the mole fraction at a constant osmotic pressure, all stable and metastable phase equilibria can be inferred graphically from the Gibbs free energy by performing common tangent constructions. In our approach, however, we merely focus on the location of the binodal and bifurcation points rather than 

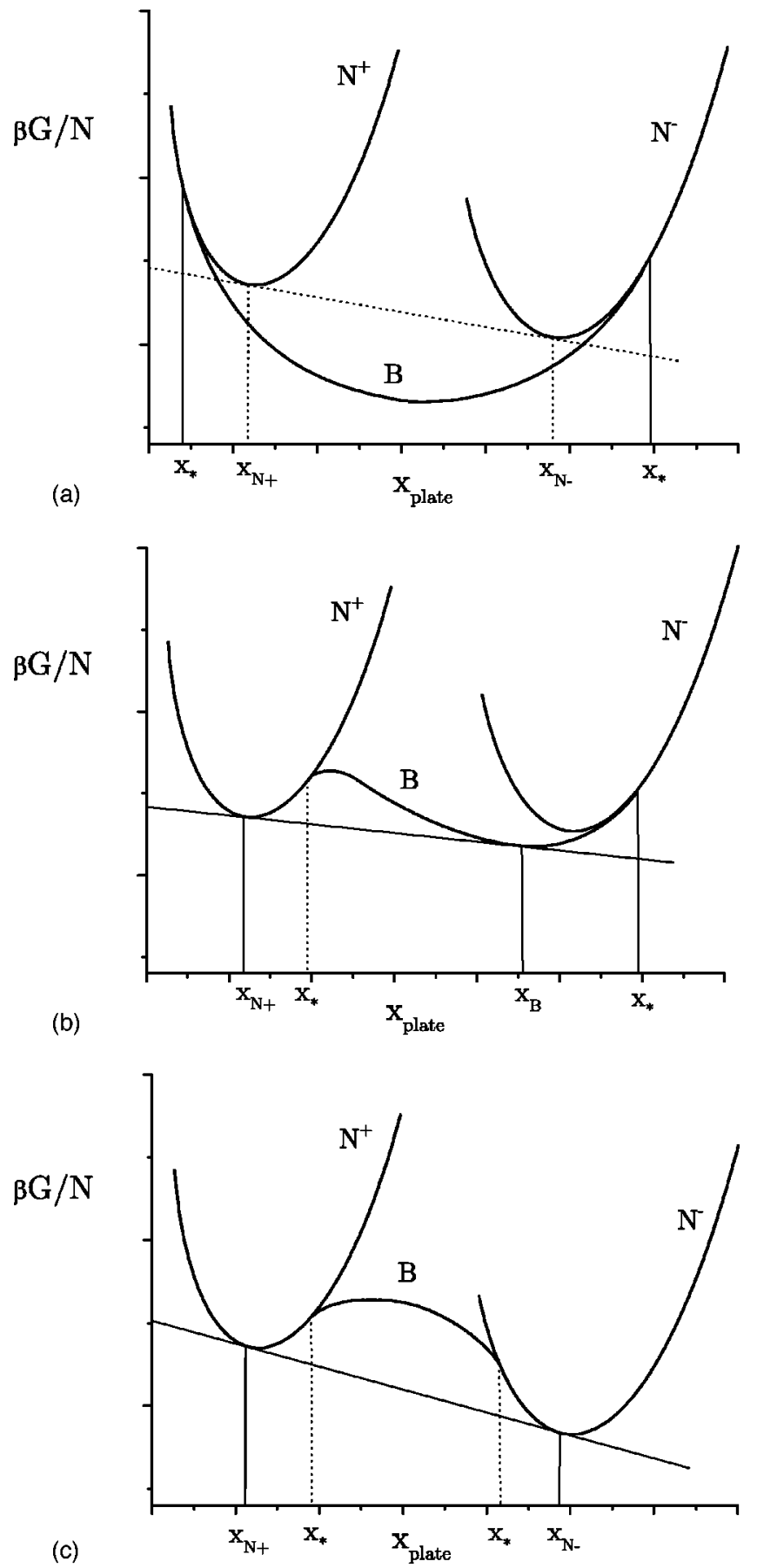

FIG. 1. Schematic illustration of the common tangent construction to determine phase coexistence in a binary rod-plate mixture. (a) Stable biaxial nematic phase, the $N^{+}-N^{-}$equilibrium is metastable (dotted lines). (b) Uniaxial-biaxial $\left(N^{+}-B\right)$ demixing. (c) Uniaxial-uniaxial $\left(N^{+}-N^{-}\right)$demixing. The biaxial nematic phase is metastable. The uniaxial-biaxial bifurcation points are indicated by $x_{*}$, all others denote binodal points. Stable phase points are indicated by solid lines, metastable ones by dotted lines.

explicitly calculating the free energy. In Fig. 1, we show that all information concerning the (meta)stability of the nematic phases can be obtained from the relative location of these points. In Fig. 1, we have sketched three scenarios. A close inspection reveals that the biaxial phase can only be stable when both uniaxial binodal points are located in between the uniaxial-biaxial bifurcation points [Fig. 1(a)]. In the opposite case [Fig. 1(c)], the biaxial nematic phase is metastable with respect to demixing into the uniaxial nematic phases. In Fig. 1(b), we have depicted a possible transitional scenario in which one bifurcation point (from the $N^{-}$phase) is located "outside" the uniaxial binodal points [as in Fig. 1(a)] while the other one lies in between. Clearly, this scenario must give rise to a stable first order uniaxial-biaxial transition $\left(N^{+}-B\right)$. Note that the $N^{+}-N^{-}$equilibria and the (second order) $N^{+}-B$ transition are both metastable in this case. We will meet this scenario in our actual calculations, but it should be mentioned that other transitional scenarios are also conceivable, depending on the exact curvature of the biaxial branch. In particular, one can think of a biaxial-biaxial demixing scenario which may occur when the $B$ branch in Fig. 1(a) displays a local maximum. However, since we choose not to calculate the Gibbs free energy of the biaxial nematic phase explicitly, the exact shape of the biaxial branch remains largely unknown. This means that we cannot completely exclude other scenarios than the ones depicted in Fig. 1 to occur in our systems.

\section{PHASE DIAGRAMS}

As mentioned in Sec. II A, the input for our phase diagram calculations are the rod-plate isotropic excluded volume ratios, $q_{12}$ and $q_{22}$, given by Eq. (7). To facilitate comparisons with the systems studied in our previous paper, we assume that the rods and plates have equal thickness, so that $L_{p}=D_{r}$. It is now convenient to rewrite Eq. (7) in terms of the particles' aspect ratios for rods $(L / D)_{R}$ and plates $(D / L)_{P}$,

$$
q_{12}=\frac{1}{4}\left(\frac{D}{L}\right)_{P}^{2} /\left(\frac{L}{D}\right)_{\mathrm{R}} \quad \text { and } \quad q_{22}=\frac{\pi}{4}\left(\frac{D}{L}\right)_{P}^{3} /\left(\frac{L}{D}\right)_{\mathrm{R}}^{2} .
$$

Henceforth, we fix the aspect ratio of the rods at $(L / D)_{R}$ $=15$, which matches the average aspect ratio of the colloidal rods used in experiment [15]. This means that we use the aspect ratio of the plates to tune the asymmetry of the mixture. Consequently, from Eq. (32) we see that the mixture is symmetric $\left(q_{22}=1\right)$ if $(D / L)_{P}=(900 / \pi)^{1 / 3} \approx 6.59$. Increasing the platelets' aspect ratio from this value will make the mixture more and more asymmetric. When $(D / L)_{P}=15$ we reach the case of the strongly asymmetric mixture studied experimentally in Ref. [15] and theoretically in Ref. [16].

\section{A. Scenario I: Stable biaxial nematic phase; bicritical point}

In Fig. 2 we show the phase diagram for the case $(D / L)_{P}=7$ which is slightly above the symmetric value. We have also constructed a volume fraction representation [Fig. 2(b)] which may be more convenient from an experimental point of view. The tie lines, which connect coexisting phases, are given by horizontal lines in the osmotic pressure representation [Fig. 2(a)] and by tilted straight lines in the volume fraction representation [Fig. 2(b)]. In the latter case, we may also draw dilution lines along which the mole fraction is kept 

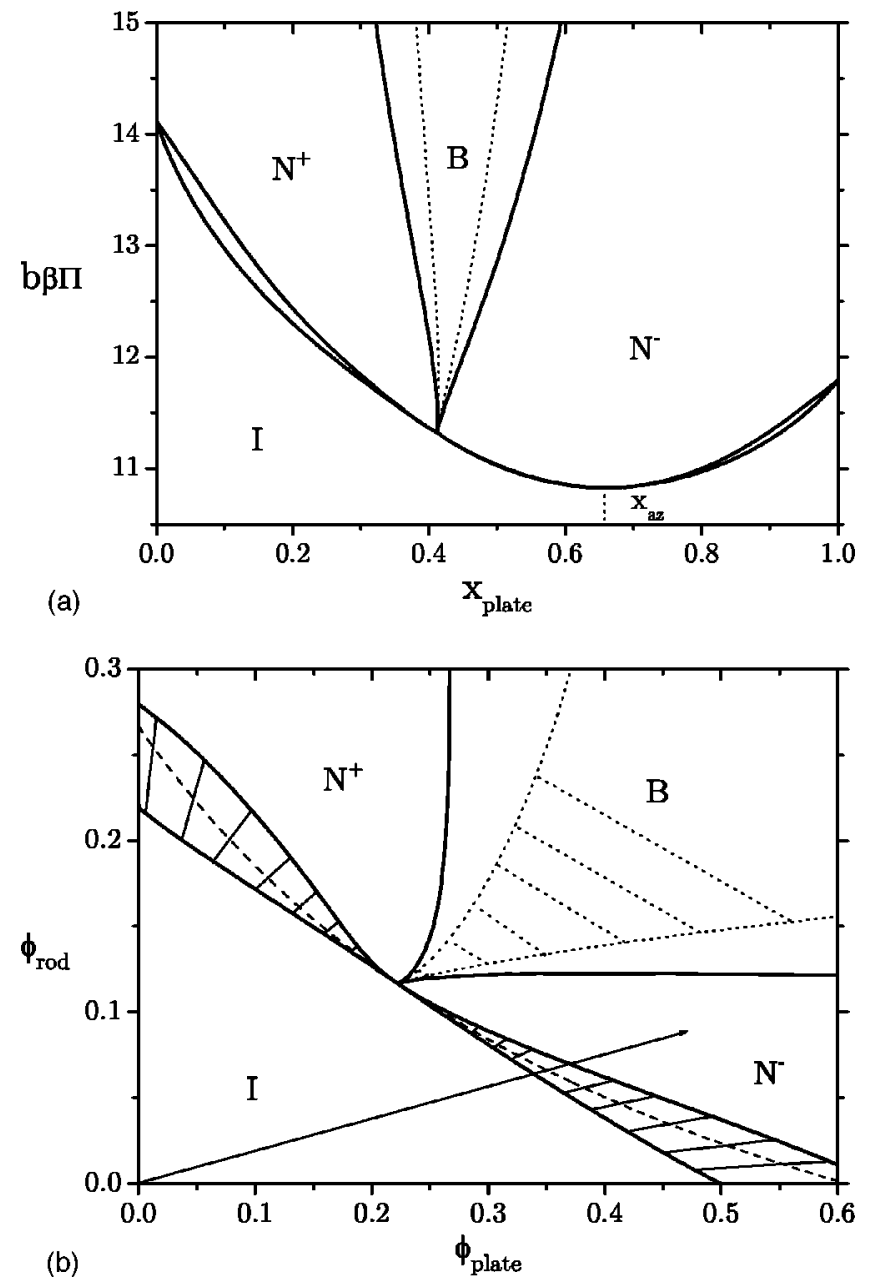

FIG. 2. (a) Scenario I: Phase diagram in the pressurecomposition plane for a slightly asymmetric mixture $\left[(D / L)_{P}\right.$ $=7]$. Thick solid lines indicate stable phase transitions. The dotted lines represent metastable $N^{+}-N^{-}$binodals. An azeotropic point is present at $x_{\mathrm{az}}=0.66$. (b) Same diagram in the volume fraction representation. Coexisting phases are connected by tilted tie lines. The dashed line represents the $I-N$ bifurcation line. The dilution line drawn corresponds to the azeotropic mole fraction.

fixed, given by straight lines running from the origin. Obviously, in the pressure representation, these dilution lines run vertically.

The topology of this diagram is very similar to the symmetric case [10]: upon compressing the system from the isotropic phase, a first order transition takes place into a uniaxial phase with the symmetry of the majority component (the rod-rich $N^{+}$phase or the plate-rich $N^{-}$phase). At higher pressures, continuous (second order) transitions from the uniaxial to the biaxial phase occur. Note that the uniaxial demixing binodals, also indicated in Fig. 2, are metastable because they lie "inside" the area marked out by the bifurcation lines. There is a special point, called a bicritical (or Landau) point where a second order transition occurs from the isotropic to the biaxial phase. In addition, the uniaxial phase boundaries come together in a sharp cusp at this point, implying that all uniaxial order parameters must go to zero there (Fig. 3). Due to the asymmetry, the bicritical point is

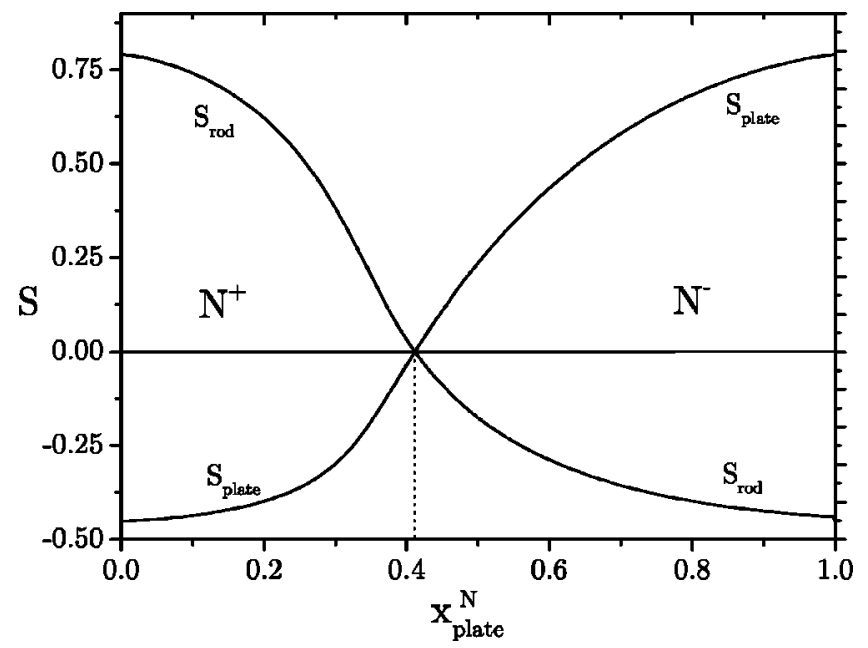

FIG. 3. Evolution of the uniaxial order parameters $S$ at $I-N$ coexistence as a function of $x_{\text {plate }}$ in the nematic phase for the case $(D / L)_{P}=7$. A critical point is located at $x_{\text {plate }}=0.41$.

now located at lower mole fractions $(x=0.41)$ compared to the symmetric case $(x=0.5)$ whereas the minimum in the osmotic pressure has shifted to higher mole fractions ( $x$ $=0.66$ ). This minimum now constitutes an azeotropic point marking equal mole fractions of the coexisting phases.

\section{B. Scenario II: Stable biaxial nematic phase; isotropic-biaxial equilibria}

Increasing the asymmetry of the mixture will eventually lead to a qualitatively different topology, as we see in Fig. 4. In this scenario, the bicritical point has disappeared which means that all transitions from the isotropic to the nematic phases have become first order. In particular, we can identify an intermediate two-phase region in which the isotropic phase coexists with the biaxial phase. The isotropic-biaxial nematic equilibria were calculated using the direct numerical solution approach, outlined in Sec. II B 2. In order to obtain reasonable quantitative results for the biaxial nematic phase, while minimizing the computational burden we used a limited grid-size $J_{\theta}=J_{\phi}=40$. To illustrate the evolution of the nematic structures along the isotropic-nematic equilibria, we have plotted the order parameters in Fig. 5. The biaxial order parameters rise from zero without a jump indicating that the structure of the coexisting nematic phase changes continuously from (rod-rich) uniaxial to biaxial back to (plate-rich) uniaxial as the mole fraction of plates is increased. The discontinuous jump around $x_{\text {plate }}^{I}=0.075$ is artificial due to the fact that we used different reference frames in the actual calculations. The phase lines were calculated starting from either a pure system of rods $(x=0)$ using the rod reference frame or a pure system of platelets adopting the plate reference frame. Note that the artificial switching from one reference frame to the other only affects the order parameters. Of course, it does not influence the thermodynamic properties of the nematic phases, as we see from the biaxial binodal in Fig. 4 , which does not show a discontinuity.

In Fig. 6, we present a detailed impression of all phase lines involved for the case $(D / L)_{P}=9.5$. From this graph we 

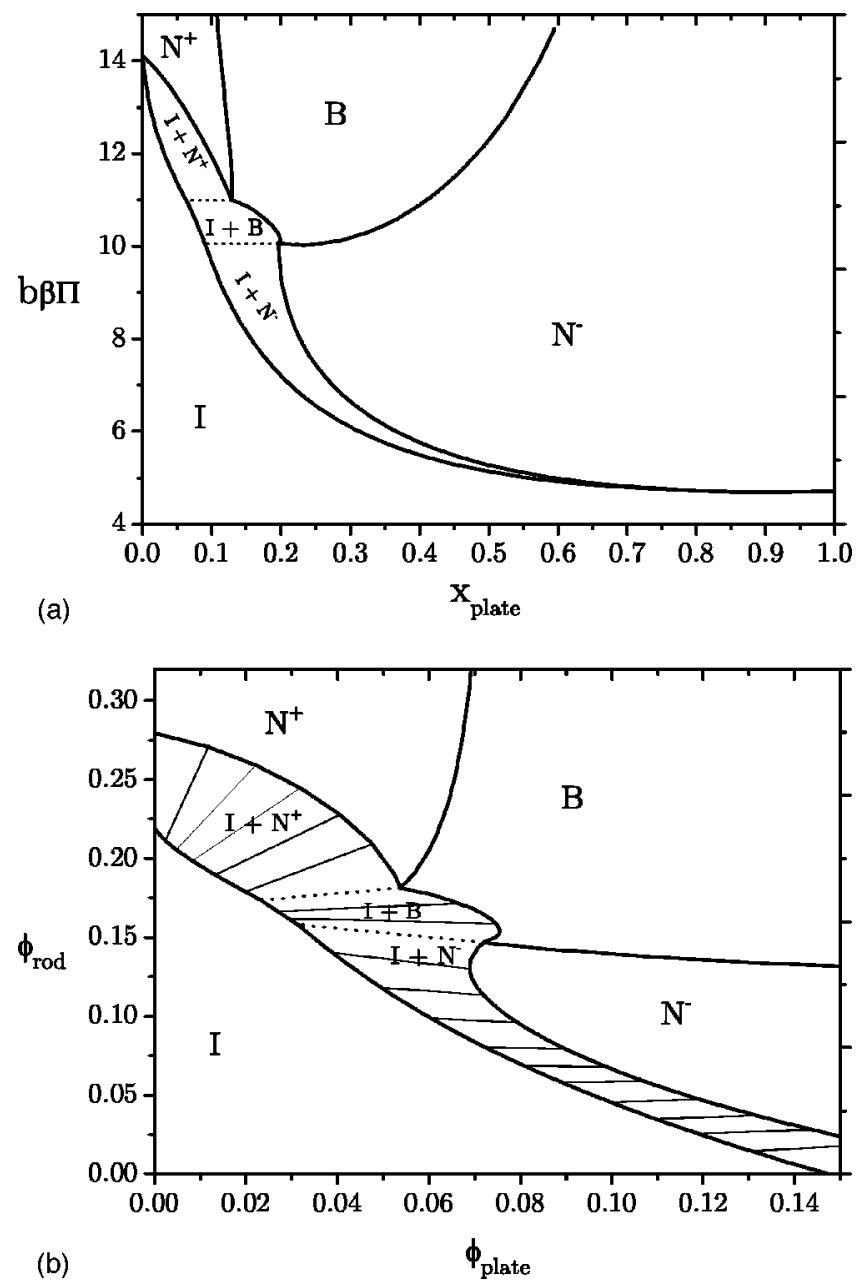

FIG. 4. (a) Scenario II: Phase diagram in the pressurecomposition plane for the case $(D / L)_{P}=9.5$. The dotted lines mark the osmotic pressures where the isotropic-nematic equilibria change continuously from uniaxial to biaxial. (b) Same diagram in the volume fraction representation. Coexisting phases are connected by tilted tie lines.

clearly see that the uniaxial demixing is still metastable with respect to the biaxial nematic phase (the $N^{+}-N^{-}$binodals run in between the bifurcation lines). The absence of a bicritical point can also be inferred from this graph; the uniaxial binodals no longer meet the bifurcation lines in a single (bicritical) point, located on the $I-N$ bifurcation line, but merge into an azeotropic end point instead. Note that the mole fractions of the uniaxial nematic phases are the same at the azeotropic point but the concentrations are not. Furthermore, the uniaxial order parameters are also nonzero at this point. Clearly, there must be a critical value for $(D / L)_{P}$ at which the bicritical point disappears by splitting into a critical point (where the $N-B$ bifurcation lines meet) and a corresponding azeotropic end point (where the uniaxial binodals meet). In Fig. 7, we have plotted the location of these points as a function of the mixture's asymmetry. The location of the biaxial critical point can easily be determined algebraically by combining the $I-N$ bifurcation equation with the $N-B$ bifurcation equation assuming the lowest degree of nematic order of the uniaxial reference phase (see the Appendix). We

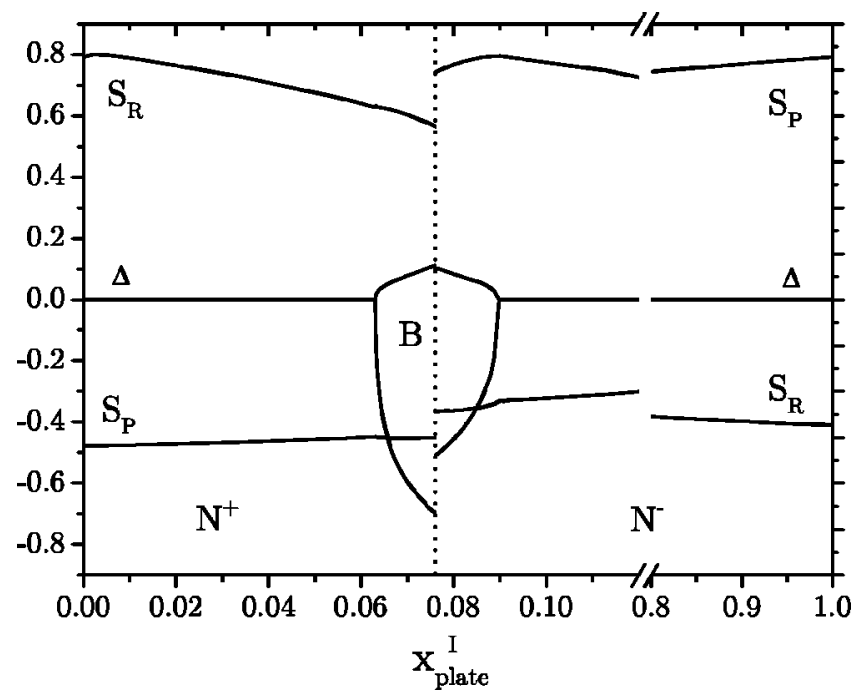

FIG. 5. Evolution of the uniaxial $(S)$ and biaxial $(\Delta)$ order parameters at isotropic-nematic coexistence as a function of $x_{\text {plate }}$ in the isotropic phase for the case $(D / L)_{P}=9.5$.

observe that the concentrations corresponding to the azeotropic end point collapse onto the curve describing the biaxial critical point at some critical value $(D / L)_{P} \approx 8$ which means that the biaxial critical point and the azeotropic end point have merged into a bicritical point. Hence we may expect a change of scenario from I to II when the aspect ratio of the platelets exceeds 8 .

\section{Scenario III: Uniaxial-biaxial demixing}

When the asymmetry is enhanced even further, the phase behavior of the rod-plate mixture changes dramatically. In Fig. 8, we have depicted the scenario for $(D / L)_{P}=14$, which is close to the experimentally accessible case $\left[(D / L)_{P}\right.$

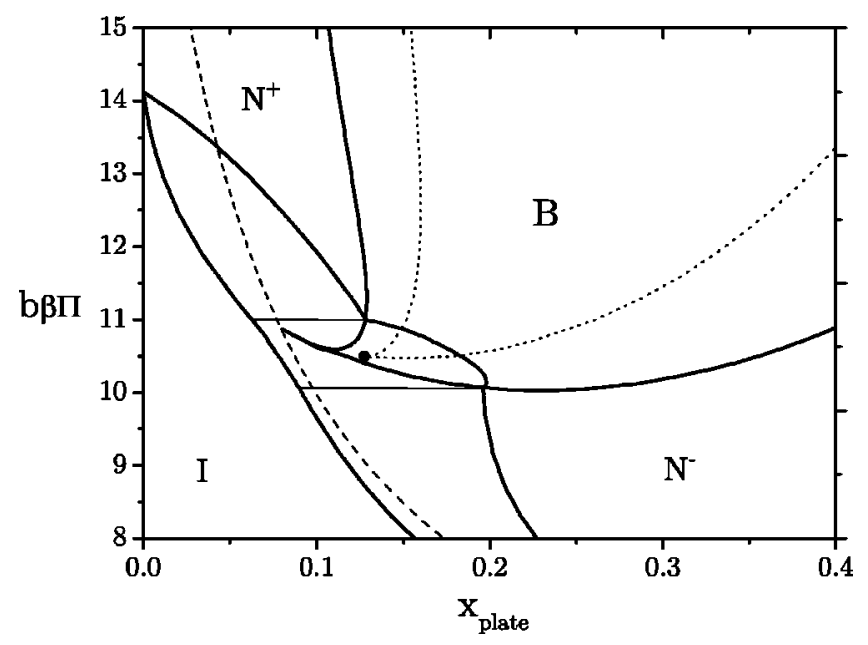

FIG. 6. Detailed picture of the phase lines for the case $(D / L)_{P}$ 9.5. Dotted lines represent metastable $N^{+}-N^{-}$binodals, the dashed curve is the $I-N$ bifurcation line. Note that the $N-B$ bifurcation lines coincide in a critical point (on the $I-N$ bifurcation line) whereas the $N^{+}-N^{-}$binodals meet in an azeotropic end point indicated by the black dot. 


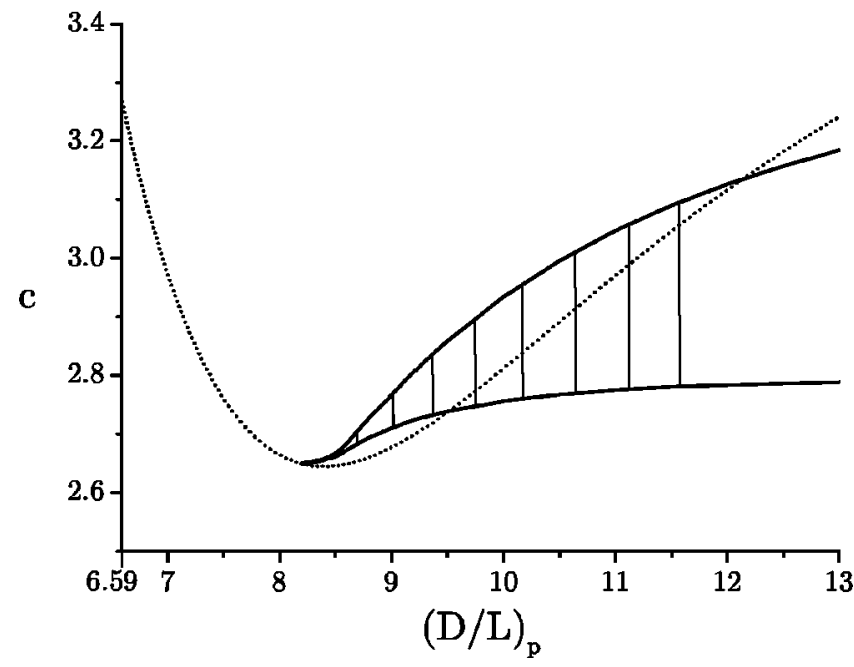

FIG. 7. Location of the biaxial critical point (dotted line) and the concentrations of the coexisting uniaxial phases of the azeotropic end point (solid lines), connected by vertical tie lines, versus $(D / L)_{P}$. At $(D / L)_{P} \lesssim 8$ all lines collapse onto a single curve, describing the location of the bicritical point.

$\left.=(L / D)_{R}=15\right]$ considered in Ref. [16]. An essential difference with the previous scenario is that a demixing occurs around $b \beta \Pi=14$ at which a rod-dominated uniaxial nematic $N^{+}$phase coexists with a biaxial phase roughly containing equal portions of each species. Furthermore, there is an associated triple point at which both $N^{+}$and $B$ coexist with an isotropic phase $I$. Upon compressing the system at higher mole fractions $(x>0.5)$ a continuous transition occurs from the plate-dominated $N^{-}$phase to the biaxial phase which subsequently demixes by splitting off a fraction of the $\mathrm{N}^{+}$ phase. Furthermore, a reentrant phenomenon is present around $x=0.4$ where the mixture displays a rich sequence of phases upon compression. To reduce computational costs, we have not explicitly calculated the isotropic-biaxial equilibria for this case but merely sketched the qualitative topology of the phase diagram at higher pressures. The justification for the demixing scenario lies in the location of the uniaxial binodals relative to the $N-B$ bifurcation lines. In Fig. $8(\mathrm{~b})$ we have displayed the Gibbs free energy at a particular osmotic pressure, in which the binodal and bifurcation points are depicted explicitly. For the sake of clarity, we have rescaled the Gibbs free energy by substracting the linear common tangent to the uniaxial branches. We see that the $N^{+}-B$ bifurcation point now has shifted to the right of the $N^{+}$binodal point whereas the $N^{-}-B$ bifurcation is still located "outside" the $N^{-}$binodal point. As already alluded to in Sec. II E, the only plausible scenario for this case is a demixing into $N^{+}$and $B$, as indicated by the sketched biaxial branch in Fig. 8(b). Note that the shape of this branch also suggests that the stable $B$ binodal point is located at slightly lower mole fractions than the metastable $N^{-}$binodal point.

To limit computational effort, we have not attempted to find the specific aspect ratio at which the $N^{+}-B$ demixing first occurs and a change of scenario from type II to III will take place. Obviously, from the results presented thus far, we know that the transition must be somewhere in the range
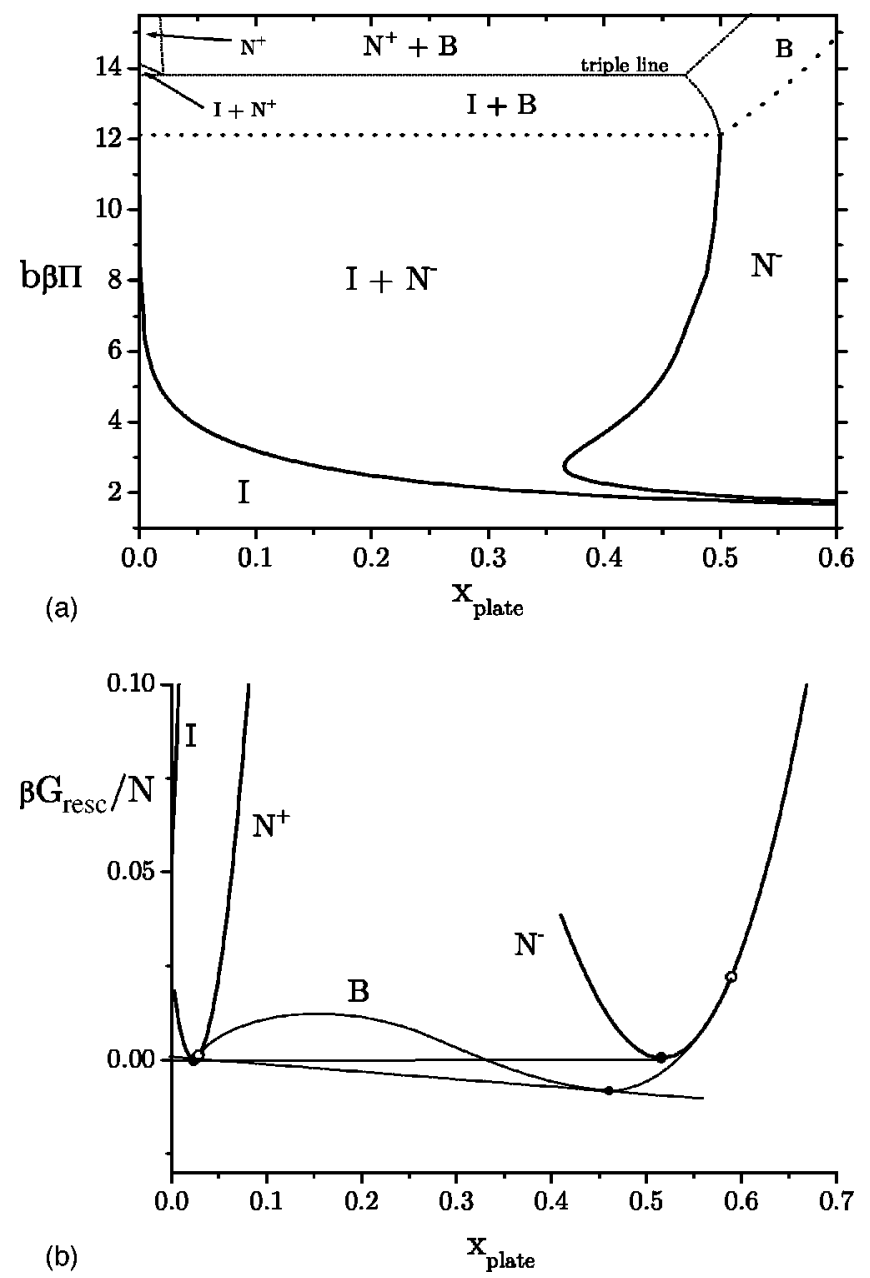

FIG. 8. (a) Scenario III: Phase diagram in the pressurecomposition plane for $(D / L)_{P}=14$. Thick solid lines indicate stable phase boundaries. The $N^{-}-B$ bifurcation line is indicated by the thick dotted line. The thin dotted lines are sketched phase lines (not calculated) outlining the qualitative phase behavior at high densities. (b) Rescaled Gibbs free energy versus $x_{\text {plate }}$ for the same mixture at constant pressure $b \beta \Pi=14.5$. Binodal and bifurcation points are indicated by black and white points, respectively. The curvature of the biaxial branch is given qualitatively by the sketched line (thin solid line). A uniaxial-biaxial $\left(N^{+}-B\right)$ demixing is evident.

$10<(D / L)_{P}<14$, which is an experimentally accessible range.

\section{Scenario IV: Uniaxial-uniaxial demixing}

Scenario III is not consistent with our previous calculations based upon the Gaussian trial function approach. In particular, the surmised demixing transition into uniaxial nematic phases, as observed experimentally and reproduced theoretically in Ref. [16] for strongly asymmetric rod-plate mixtures is not found in our numerical analysis of the EulerLagrange equations. Instead, we observe a demixing into a rod-rich uniaxial nematic phase and a biaxial nematic phase (containing approximately 50\% platelets) for a mixture of rods and plates with aspect ratios around 15 . The question now arises whether or not a demixing into the uniaxial nem- 

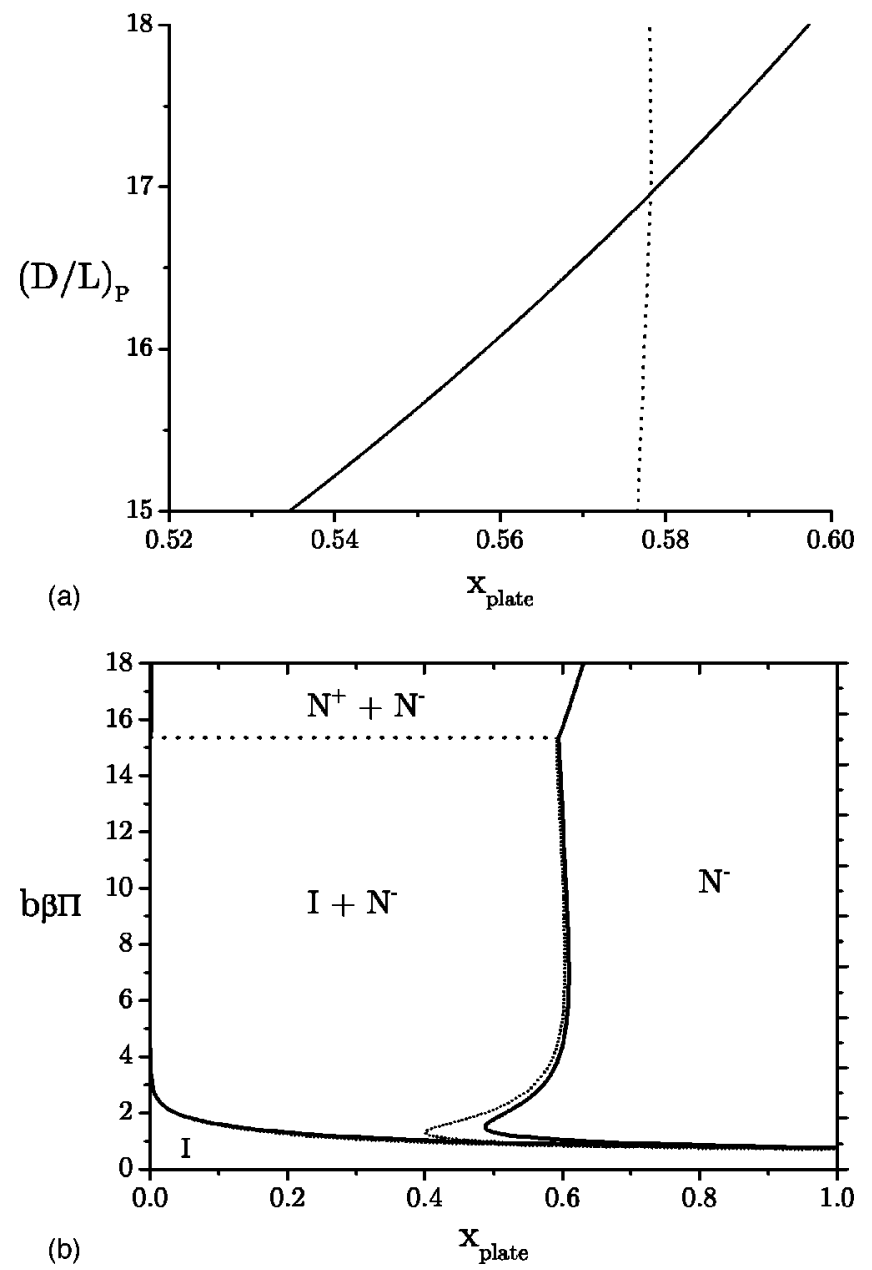

FIG. 9. (a) Position of the $N^{-}$binodal point (in terms of $x_{\text {plate }}$ ) relative to the $N^{-}-B$ bifurcation point at constant pressure $b \beta \Pi$ $=14.12$ for various $(D / L)_{P}$. Beyond the intersection [around $\left.(D / L)_{p}=17\right]$ the biaxial nematic phase becomes fully metastable. (b) Scenario IV: Phase diagram in the pressure-composition plane for $(D / L)_{P}=18$ calculated within the Gaussian approximation. The $I-N^{+}-N^{-}$triple line is indicated by the horizontal dotted line. The dotted curves represent the numerically exact $I-N^{-}$binodals.

atic phases (scenario IV) is recovered when the aspect ratio of the platelets is increased beyond 15. Considering Fig. $8(\mathrm{~b})$, one can imagine that, upon increasing $(D / L)_{P}$, the asymmetry may force the $N^{-}-B$ bifurcation point to shift in between the uniaxial binodal points such that the biaxial nematic phase becomes fully metastable with respect to a $N^{+}-N^{-}$demixing, according to Fig. 1(b). To verify this possibility we have calculated the location of these points at a fixed osmotic pressure, namely, the $I-N$ coexistence pressure for the pure system $(b \beta \Pi=14.12)$ which is slightly above the triple pressure. In Fig. 9(a) we have depicted the evolution of the $N^{-}$binodal (corresponding to the $I-N^{-}$ equilibria) and the $N^{-}-B$ bifurcation point as a function of $(D / L)_{P}$. The intersection point around $(D / L)_{P}=17$ reveals that there must be a scenario IV such that the uniaxial demixing is indeed recovered, albeit at a higher plate aspect ratio than expected from Ref. [16]. For the sake of completeness, we have depicted the phase diagram for the case
$(D / L)_{P}=18$ in Fig. 9(b), in which calculations were based upon the Gaussian trial function approach, as discussed in detail in Ref. [16]. Note that this diagram is qualitatively the same as the one presented in Ref. [16] although the $N^{+}$and $I-N^{+}$coexistence regions are hardly visible in Fig. 9(b) due to the extremely low mole fractions of the coexisting $I$ and $N^{+}$phases. Comparison with the numerical exact $I-N^{-}$binodals shows that the Gaussian approximation provides increasingly better quantitative results at high compressions where the alignment of the particles (in both polar and planar direction) is particularly strong. Deviations occur at lower osmotic pressure, in particular around $b \beta \Pi=2$, showing that the reentrant phenomenon is underestimated somewhat by the Gaussian approximation.

\section{SUMMARY AND CONCLUSIONS}

We have investigated the role of the rod-plate excluded volume ratio $\left(v_{\mathrm{ex}}^{\mathrm{pp}} / v_{\mathrm{ex}}^{\mathrm{rr}}\right)$ in the phase behavior of asymmetric mixtures of cylindric rods and platelets (for which $v_{\mathrm{ex}}^{\mathrm{pp}}$ $>v_{\mathrm{ex}}^{\text {rr }}$ ) using a simple Onsager type density functional theory. The phase diagrams were calculated from an exact numerical analysis of the Euler-Lagrange equations, obtained from formally minimizing the free energy, such that no simplifications were made $a$ priori with respect to the ODF. Our particular interest was focussed on the stability of the biaxial nematic phase in relation to the mixture's asymmetry. Starting from the symmetric case we enhanced the asymmetry of the mixture by varying the platelet aspect ratio in the range $7<(D / L)_{P}<18$ while keeping the rod aspect ratio fixed at 15. Considering the role of the biaxial nematic phase in the overall topology of the phase diagram, we were able to distinguish four scenarios.

Upon increasing the plate aspect ratio from its symmetric value (6.59), we observe that the characteristic bicritical point is retained initially (scenario I) but disappears around $(D / L)_{P}=8$ and is replaced by a two-phase region marking first order transitions from the isotropic to the biaxial nematic phase (scenario II). At higher asymmetries [around $\left.(D / L)_{P}=15\right]$ we found a uniaxial-biaxial $\left(N^{+}-B\right)$ demixing transition with an associated $I-N^{+}-B$ triple equilibrium (scenario III). Increasing the aspect ratio beyond 17 will give a uniaxial-uniaxial $\left(N^{+}-N^{-}\right)$demixing with an associated $I-N^{+}-N^{-}$triple point (scenario IV). This indicates that the biaxial nematic phase may become fully metastable in highly asymmetric mixtures. To limit the computational burden, we have not explicitly calculated the isotropic-biaxial $(I-B)$ and uniaxial-biaxial $\left(N^{+}-B\right)$ equilibria for scenario III. Therefore, it should be noted that, due to the uncertainty in the thermodynamic properties of the biaxial phase, other scenarios than the ones presented in this paper cannot be completely ruled out. Nevertheless, we believe that our scenarios are sufficiently plausible.

There is experimental evidence of the uniaxial-uniaxial demixing transition (scenario IV) to occur in mixtures of colloidal rods and platelets, albeit at a slightly lower plate aspect ratio (of roughly 15). However, no detailed structure investigation on the nematic phases has been performed in Ref. [15], so that there are no conclusive results available as 
to whether the demixed nematic phases are really uniaxial or possibly have some degree of biaxiality. Therefore, considering our present theoretical predictions, it would be intriguing to verify the possibility of a uniaxial-biaxial demixing scenario to occur in these experimental systems. Of course, this would require a thorough reexamination of the experimental systems focusing on the optical properties of the nematic textures (particularly, the plate-dominated nematic phase). Furthermore, our results also suggest that the formation of a biaxial nematic phase can be promoted experimentally by decreasing the diameter of the colloidal platelike colloids, thereby reducing the mixture's asymmetry. However, it should be noted that the effect of polydispersity and the influence of higher-order particle correlations (both are not incorporated here) may give rise to qualitatively different scenarios from the ones predicted by our calculations.

\section{APPENDIX}

\section{Calculation of the biaxial critical point}

Figure 6 shows that the $N-B$ bifurcation lines emanate from the $I-N$ bifurcation line at a critical point where the order parameters are necessarily zero. To calculate this point for a given asymmetry, we may perform a $N-B$ bifurcation analysis starting from a weakly ordered uniaxial phase. Assuming the lowest degree of nematic order in the uniaxial reference phase, we may approximate the uniaxial ODFs by Eq. (22). Substitution into Eq. (28) yields for the coefficients $W_{11}^{(j)}$,

$$
\begin{aligned}
W_{11}^{(j)} & =\frac{1}{4 !} \int_{0}^{1}\left[P_{2}^{2}(t)\right]^{2}\left[1+\alpha_{2}^{(j)} P_{2}(t)\right] d t \quad(t=\cos \theta) \\
& =\frac{1}{5}-\frac{2}{7} S_{j},
\end{aligned}
$$

in terms of the uniaxial order parameters $S_{j}=\alpha_{2}^{(j)} / 5$. Using this together with $d_{2}=-5 \pi / 32$ and $c_{2}=5 / 8$ [from Eq. (12)] we obtain, from Eq. (29), the following linear set:

$$
\begin{aligned}
\beta_{2}^{2(1)}= & \frac{c}{4}\left[(1-x)\left(1-\frac{10}{7} S_{1}\right) \beta_{2}^{2(1)}\right. \\
& \left.-2 x q_{12}\left(1-\frac{10}{7} S_{2}\right) \beta_{2}^{2(2)}\right],
\end{aligned}
$$

$$
\begin{aligned}
\beta_{2}^{2(2)}= & \frac{c}{4}\left[-2(1-x) q_{12}\left(1-\frac{10}{7} S_{1}\right) \beta_{2}^{2(1)}\right. \\
& \left.+x q_{22}\left(1-\frac{10}{7} S_{2}\right) \beta_{2}^{2(2)}\right] .
\end{aligned}
$$

Setting $S_{j}=0$ in Eq. (A2) leads to the characteristic equation for the $I-N$ bifurcations Eq. (25) implying that the isotropicbiaxial bifurcation concentrations are the same as the isotropic-uniaxial bifurcation densities, for any mole fraction [9].

Since the bicritical point must be a solution of Eq. (25), we may subtract Eq. (25) from the characteristic equation corresponding to (A2) to get the following equation:

$$
\begin{gathered}
(1-x) S_{1}+x q_{22} S_{2}+\frac{c}{4} x(1-x)\left(4 q_{12}^{2}-q_{22}\right) \\
\times\left[S_{1}+S_{2}-\frac{10}{7} S_{1} S_{2}\right]=0 .
\end{gathered}
$$

Ignoring the $O\left(S^{2}\right)$ term and eliminating $S_{j}$ using the relation

$$
S_{2}=S_{1} \frac{\left[\frac{c}{2}(1-x)-2\right]}{c x q_{12}},
$$

from Eq. (23), we obtain

$$
\begin{aligned}
q_{22} x & +\frac{c}{4} x(1-x)\left\{\left[4 q_{12}^{2}-2\left(q_{12}+q_{22}\right)\right]\right. \\
- & \left.\frac{c}{4}\left[4 q_{12}^{2}-q_{22}\right]\left[2 x q_{12}+(1-x)\right]\right\}=0 .
\end{aligned}
$$

Solving this equation together with the $I-N$ bifurcation equation (25) will uniquely determine the biaxial critical point (in terms of $x$ and $c$ ) for any given set of parameters $q_{j k}$. The solutions for the symmetric case, discussed in Ref. [6], can be recovered by substituting $x=1 / 2$ and $q_{22}=1$ in Eq. (A5) to obtain $c=8 /\left(2 q_{12}+1\right)$ and $S_{1}=-S_{2}$.
[1] H. Zocher, Z. Anorg. Allg. Chem. 147, 91 (1925).

[2] I. Langmuir, J. Chem. Phys. 6, 873 (1938).

[3] L. Onsager, Ann. N.Y. Acad. Sci. 51, 627 (1949).

[4] J.D. Bernal and I. Fankuchen, J. Gen. Physiol. 25, 111 (1941).

[5] H.N.W. Lekkerkerker, P. Coulon, R. van der Hagen, and R. Deblieck, J. Chem. Phys. 80, 3427 (1984); R. Deblieck and H.N.W. Lekkerkerker, J. Phys. (France) Lett. 41, L-351 (1980).

[6] A. Stroobants and H.N.W. Lekkerkerker, J. Phys. Chem. 88, 3669 (1984).
[7] Y. Rabin, W.E.M. Mullen, and W.M. Gelbart, Mol. Cryst. Liq. Cryst. 89, 67 (1982).

[8] P.J. Camp and M.P. Allen, Physica A 229, 410 (1996).

[9] A. Chrzanowska, Phys. Rev. E 58, 3229 (1998).

[10] S. Varga, A. Galindo, and G. Jackson, Phys. Rev. E 66, 011707 (2002).

[11] R. Alben, J. Chem. Phys. 59, 4299 (1973).

[12] R. van Roij and B. Mulder, J. Phys. II 4, 1763 (1994).

[13] R. Zwanzig, J. Chem. Phys. 39, 1714 (1963).

[14] P.J. Camp, M.P. Allen, P.G. Bolhuis, and D. Frenkel, J. Chem. 
Phys. 106, 9270 (1997).

[15] F.M. van der Kooij and H.N.W. Lekkerkerker, Phys. Rev. Lett. 84, 781 (2000); Langmuir 44, 10144 (2000).

[16] H.H. Wensink, G.J. Vroege, and H.N.W. Lekkerkerker, J. Chem. Phys. 115, 7319 (2001).

[17] A. Speranza and P. Sollich, J. Chem. Phys. 117, 5421 (2002).
[18] R.F. Kayser and H.J. Raveche, Phys. Rev. A 17, 2067 (1978).

[19] K. Lakatos, J. Stat. Phys. 2, 121 (1970).

[20] I.S. Gradshteyn and I.M. Ryzhik, Table of Integrals, Series and Products (Academic Press, San Diego, 1994).

[21] J. Herzfeld, A.E. Berger, and J.W. Wingate, Macromolecules 17, 1718 (1984). 